Bull. Mater. Sci., Vol. 36, No. 3, June 2013, pp. 345-351. (c) Indian Academy of Sciences.

\title{
Exploring a novel approach to fabricate vanadium carbide encapsulated into carbon nanotube (VC@C) with large specific surface area
}

\author{
YIFU ZHANG ${ }^{\mathrm{a}}$, JUECHENG ZHANGa ${ }^{\mathrm{a}}$ MEIJUAN FAN ${ }^{\mathrm{b}}$ ，YAN'AN LONG ${ }^{\mathrm{a}}$, YALAN ZHONG ${ }^{\mathrm{a}}$, \\ XINGHAI LIU ${ }^{\mathrm{b}}$ and CHI HUANG ${ }^{\mathrm{a}, \mathrm{c}, *}$ \\ ${ }^{a}$ College of Chemistry and Molecular Sciences, Wuhan University, Wuhan 430 072, P. R. China \\ ${ }^{\mathrm{b}}$ School of Printing and Packaging, Wuhan University, Wuhan 430 079, P. R. China \\ ${ }^{\mathrm{c}}$ Engineering Research Centre of Organosilicon Compound and Material, Ministry of Education of China, \\ Wuhan 430 072, P. R. China
}

MS received 9 March 2012; revised 26 April 2012

\begin{abstract}
A novel approach to the fabrication of vanadium carbide encapsulated into carbon nanotube (VC@C) core-shell structured composite by thermal treatment with the precursor $\mathrm{V}_{3} \mathrm{O}_{7} \cdot \mathrm{H}_{2} \mathrm{O} @ \mathrm{C}$ was developed for the first time. The as-obtained VC@C were characterized by X-ray powder diffraction (XRD), Raman spectrum, energydispersive X-ray spectrometer (EDX), elemental analysis (EA), Fourier transform infrared spectroscopy (FT)(IR), scanning electron microscopy (SEM), transmission electron microscopy (TEM) and Brunauer-Emmett-Teller (BET). The results showed that VC@C with core-shell structures could be successfully synthesized at $1000{ }^{\circ} \mathrm{C}$ for $2 \mathrm{~h}$. The specific surface area, average pore size and measured pore volume of VC@C were $135.46 \mathrm{~m}^{2} / \mathrm{g}, 4.443 \mathrm{~nm}$ and $0.180 \mathrm{~cm}^{3} / \mathrm{g}$, respectively indicating that the as-obtained VC@C composite could be used as a mesoporous material. Furthermore, thermal behaviour of the as-obtained VC@C composite in air was investigated by thermogravimetric/differential thermal analyser (TG/DTA). The experimental result revealed that the carbon coated on the surface of $\mathrm{VC}$ has high activity with $\mathrm{O}_{2}$ in air atmosphere.
\end{abstract}

Keywords. VC@C; core-shell structure; composite materials; mesoporous material; solid-state reaction.

\section{Introduction}

The fabrication of new materials with well-defined morphologies based on the construction of some other materials with novel structures has gained considerable attention in the past few years (Zygmunt et al 2003; Zhang et al 2011a, 2012a, b). For example, novel silica nanotubes with high aspect ratio were synthesized using $\mathrm{V}_{3} \mathrm{O}_{7} \cdot \mathrm{H}_{2} \mathrm{O}$ nanofibres as templates by Zygmunt et al (2003) and the silica nanotubes had been found a novel use in coating with catalytically active transition metal compounds (Zygmunt et al 2003). Recently, core-shell nanostructures have been paid much attention due to their tunable surface properties, which retain the properties of both cores and shells (Caruso 2001; Sun et al 2006; Singh et al 2010; Zhang et al 2011b, c). Moreover, core-shell structured materials with a carbon shell have stimulated great interest, owing to their chemical inertness, biocompatibility and high thermal conductivity (Sun and Li 2004; Dastidar et al 2011; Zhang et al 2011b, c, 2012d, e). It has been reported that $\mathrm{V}_{2} \mathrm{O}_{5}$ can react with active carbon to synthesize low-valence vanadium oxides and vanadium carbide (Gupta and Krishnamurthy 1992 (chapter 4)). The idea that low-valence vanadium oxides@C or VC@C and core-shell structures are

\footnotetext{
*Author for correspondence (chihuang@whu.edu.cn)
}

synthesized using high-valence vanadium oxides@C and core-shell structures have not been reported to the best of our knowledge, which is a novel route to fabricate new materials with novel structures and morphologies.

In the past decades, the transition metal carbides have attracted a great interest owing to their outstanding physical and chemical properties, including high melting point, high thermal conductivity, high hardness, high strength even at high temperatures, high chemical resistance, etc which have been extensively applied in metallurgy, cutting tools, electronics, catalysts, high-temperature structural materials, and so on (Oelerich et al 2001; Yoon et al 2002; Liermann et al 2004; Reichl and Hayek 2004; Yazawa et al 2004; Nakamura and Yashima 2008; Ye et al 2010; Fard and Baharvandi 2011; Pang et al 2011). Among them, vanadium carbide (VC) is a very important industrial material because of its excellent high-temperature strength, high chemical and thermal stability even at high temperatures. Therefore, VC can be widely applied to cutting materials, abrasive and anti-wear materials (Calka and Kaczmarek 1992; Ma et al 2009; Chen et al 2011). Furthermore, vanadium carbides own catalytic behaviours, which are almost comparable to platinum metal due to their similar electronic and magnetic properties (Choi 1999; Frantz et al 2002; Rodriguez et al 2004; Chen et al 2011). Recently, Hu's group reported (Hu et al 2011) that the oxygen reduction 
electrocatalysis can be enhanced by nano-sized cubic vanadium carbide. Therefore, developing a novel route to synthesize nanocrystalline VC is greatly meaningful and a crucial challenge for material scientists (Chen et al 2011; Hu et al 2011).

Up to now, various methods have been reported to prepare vanadium carbide and some approaches have been widely used for its production, such as, high temperature reactions (Gupta and Krishnamurthy 1992), direct element reaction (Schwarzkopf and Kieffer 1953), mechanical alloying (Zhang and Li 2005), gas reduction-carburization (Lee et al 1990), temperature-programmed reaction (Kapoor and Oyama 1995), thermal processing of the precursor with additives (Ma et al 2009; Zhao et al 2009; Ye et al 2010), aluminothermic reduction of vanadium oxide (Venugopalan and Sathiyamoorthy 2006), the carburization of vanadium oxide with an organic reagent (Li et al 2008), etc. Recently, flowerlike vanadium carbide hierarchical nanocrystals were synthesized by the hydrothermal process using a mixture of diethanolamine $\left(\mathrm{HN}\left(\mathrm{C}_{2} \mathrm{H}_{4} \mathrm{OH}\right)_{2}\right.$, DEA) and $\mathrm{V}_{2} \mathrm{O}_{5}$ powder reported by Ma et al (2010). However, the synthesis of VC encapsulated into carbon nanotube (VC@C core-shell structured composite) has not been reported to the best of our knowledge.

Herein, we have successfully developed a novel approach to fabricate VC@C core-shell composite by thermal treatment with the precursor $\mathrm{V}_{3} \mathrm{O}_{7} \cdot \mathrm{H}_{2} \mathrm{O} @ \mathrm{C}$ for the first time. The as-obtained VC@C has large specific surface area and lots of pores, indicating that it can be used as a mesoporous material. Furthermore, thermal behaviour of VC@C in air was investigated by TG-DTA test, and it was found that the carbon coated on the surface of $\mathrm{VC}$ has high activity with $\mathrm{O}_{2}$ in an air atmosphere. The above results indicate that VC@C may have high catalytic activity and have potential application in the future.

\section{Experimental}

\subsection{Materials}

Vanadium pentoxide $\left(\mathrm{V}_{2} \mathrm{O}_{5}\right)$, ethanol and $\mathrm{D}$-(+)-glucose $\left(\mathrm{C}_{6} \mathrm{H}_{12} \mathrm{O}_{6} \cdot \mathrm{H}_{2} \mathrm{O}\right)$ with analytical grade were purchased from Sinopharm Chemical Reagent Co. Ltd. and used without any further purification.

\subsection{Synthesis of $\mathrm{V}_{3} \mathrm{O}_{7} \cdot \mathrm{H}_{2} \mathrm{O}$ nanobelts}

The synthesis of $\mathrm{V}_{3} \mathrm{O}_{7} \cdot \mathrm{H}_{2} \mathrm{O}$ nanobelts was based on our previous report (Zhang et al 2010) and slightly modified. In a typical synthesis, $9 \mathrm{~g}$ of $\mathrm{V}_{2} \mathrm{O}_{5}$ powder was dispersed into $30 \mathrm{~mL}$ of ethanol and then $370 \mathrm{~mL}$ of deionized water was added into the above solution with magnetic stirring. The mixed solution was transferred into a $600 \mathrm{~mL}$ stainless steel autoclave after the solution became suspension. An autoclave was sealed and maintained at $180{ }^{\circ} \mathrm{C}$ for $48 \mathrm{~h}$ and then cooled to room temperature naturally. The products were filtered off, washed with distilled water and absolute ethanol several times to remove all possible residues and dried in vacuum at $75{ }^{\circ} \mathrm{C}$ for the following experiments. XRD pattern of the as-obtained product is shown in supplementary data (figure S1)

\subsection{Synthesis of $\mathrm{V}_{3} \mathrm{O}_{7} \cdot \mathrm{H}_{2} \mathrm{O} @ \mathrm{C}$ core-shell composite}

The synthesis of $\mathrm{V}_{3} \mathrm{O}_{7} \cdot \mathrm{H}_{2} \mathrm{O} @ \mathrm{C}$ core-shell composite was similar with our previous reports (Zhang et al 2011c). In a typical procedure, $0.40 \mathrm{~g}$ of the as-obtained $\mathrm{V}_{3} \mathrm{O}_{7} \cdot \mathrm{H}_{2} \mathrm{O}$ nanobelts were dispersed into the glucose solution $(4 \mathrm{~g}$ of glucose and $50 \mathrm{~mL}$ of distilled water) in a $100 \mathrm{~mL}$ beaker under ultrasonic for $20 \mathrm{~min}$ and then the mixture was stirred vigorously for $1 \mathrm{~h}$ by magnetic stirrer. After the solution became suspension, they were transferred into a $100 \mathrm{~mL}$ teflon-lined stainless steel autoclave, which was sealed and maintained at $180{ }^{\circ} \mathrm{C}$ for $4 \mathrm{~h}$. After cooling to room temperature naturally, the products were filtered off, washed with distilled water and absolute ethanol several times and dried in vacuum at $75{ }^{\circ} \mathrm{C}$ for further characterization and application. XRD pattern of the as-obtained $\mathrm{V}_{3} \mathrm{O}_{7} \cdot \mathrm{H}_{2} \mathrm{O} @ \mathrm{C}$ is shown in supplementary data (figure S1).

\subsection{Synthesis of VC@C core-shell material}

To obtain VC@C core-shell material, the above core-shell structured composite $\left(\mathrm{V}_{3} \mathrm{O}_{7} \cdot \mathrm{H}_{2} \mathrm{O} @ \mathrm{C}\right)$ was heated in a tube furnace with $5{ }^{\circ} \mathrm{C} / \mathrm{min}$ heating rate under a flow of argon gas at $700-1000{ }^{\circ} \mathrm{C}$ for $2 \mathrm{~h}$ and cooled to room temperature in an argon flow to prevent oxidation of VC@C composite.

\subsection{Characterization}

X-ray powder diffraction (XRD) was carried out on D8 $\mathrm{X}$-ray diffractometer equipment with $\mathrm{CuK} \alpha$ radiation, $\lambda=$ $1.54060 \AA$. The Raman spectrum was taken on a RM-1000 spectrometer (confocal Raman microspectroscopy) with an argon-ion laser at an excitation wavelength of $514.5 \mathrm{~nm}$. The elemental analysis (EA) of the samples was carried out using a Vario EL III equipment (Germany) with a TCD detector to analyse the element of $\mathrm{C}, \mathrm{H}$ and $\mathrm{N}$. The chemical composition of as-obtained samples was revealed by the energy-dispersive X-ray spectrometer (EDX) attached to a scanning electron microscope (SEM; Quanta 200). Fourier transform infrared spectroscopy (FT-IR) pattern of the solid samples was measured using $\mathrm{KBr}$ pellet technique (about $1 \mathrm{wt} \%$ of the samples and $99 \mathrm{wt} \%$ of $\mathrm{KBr}$ were mixed homogeneously and then the mixture was pressed to a pellet) and recorded on a Nicolet $60-\mathrm{SXB}$ spectrometer from 4000 to $400 \mathrm{~cm}^{-1}$ with a resolution of $4 \mathrm{~cm}^{-1}$. The morphology and dimension of the products were observed by scanning electron microscopy (SEM, Quanta 200) and transmission electron microscopy (TEM, JEM-100CXII). The specific surface area and mesoporous properties of the as-obtained sample were examined by Brunauer-Emmett-Teller (BET) $\mathrm{N}_{2}$-gas 

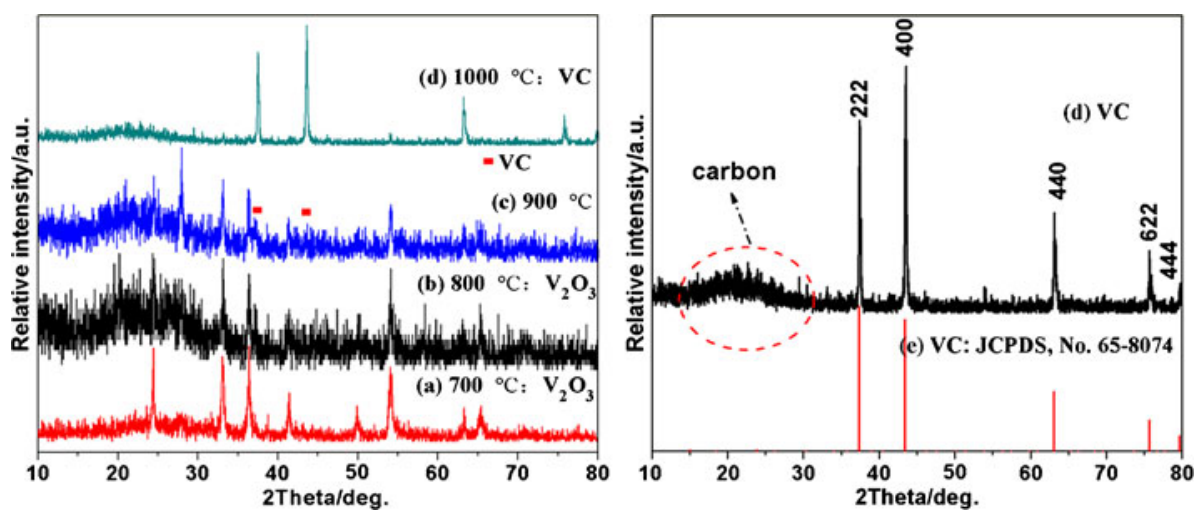

Figure 1. XRD patterns of resulting products obtained with different heating temperatures for 2 h: (a) $700{ }^{\circ} \mathrm{C}$; (b) $800{ }^{\circ} \mathrm{C}$; (c) $900{ }^{\circ} \mathrm{C}$; (d) $1000{ }^{\circ} \mathrm{C}$ and (e) plots of JCPDS No. 65-8074.

adsorption method at $77 \mathrm{~K}$ and carried out on a JW-BK equipment. Pore volume and average pore size were calculated by Barrett-Joyner-Halenda (BJH) formula. Thermogravimetric/differential thermal analyser (TG/DTA) was performed on SETSYS-1750 (AETARAM instruments). About $10 \mathrm{mg}$ of the as-obtained samples was heated in an $\mathrm{Al}_{2} \mathrm{O}_{3}$ crucible in nitrogen atmosphere from ambient temperature to $800{ }^{\circ} \mathrm{C}$ at a constant rise of temperature $\left(10^{\circ} \mathrm{C} / \mathrm{min}\right)$.

\section{Results and discussion}

Figure 1 shows typical XRD patterns of the resulting products obtained by different treatment temperatures for $2 \mathrm{~h}$. All the peaks from figure 1(a and b) can be indexed to the rhombohedral crystalline phase (space group: $R-3 c 167)$ of $\mathrm{V}_{2} \mathrm{O}_{3}$, which corresponds to the literature values (JCPDS No. 34-0187, 1984). The above result indicated that pure $\mathrm{V}_{2} \mathrm{O}_{3}$ was synthesized when the heating temperatures range was $700-800{ }^{\circ} \mathrm{C}$. However, a new phase is observed from the XRD pattern (figure 1(c)) with the temperature up to $900{ }^{\circ} \mathrm{C}$. Consequently, new phase with high purity is prepared by increasing the thermal temperature to $1000{ }^{\circ} \mathrm{C}$, whose XRD observation is represented in figure 1(d). All the diffraction peaks in figure 1 (d) can be assigned to the cubic phase (space group: $F m-3 m$, No. 225) of VC with calculated lattice parameters $a=4.165 \AA$, which are in agreement with the literature values (JCPDS No. 65-8074, $a=$ $4.164 \AA$ ) (Pessall et al 1968), whose plots are shown in figure 1(e). No peaks of any other phases are detected from the XRD pattern, indicating the as-prepared VC with high purity. Furthermore, we can also see a broadened peak ranging from 15 to $30^{\circ}$, which is attributed to amorphous carbon (Sun et al 2006; Zhang et al 2011b). These results reveal that VC@C core-shell composite is successfully fabricated.

According to our previous report, $\mathrm{V}_{3} \mathrm{O}_{7} \cdot \mathrm{H}_{2} \mathrm{O}$ loses water at about $380{ }^{\circ} \mathrm{C}$ (Zhang et al 2010) and the equation is presented as (1):

$$
\mathrm{V}_{3} \mathrm{O}_{7} \cdot \mathrm{H}_{2} \mathrm{O} \rightarrow \mathrm{V}_{3} \mathrm{O}_{7}+\mathrm{H}_{2} \mathrm{O} .
$$

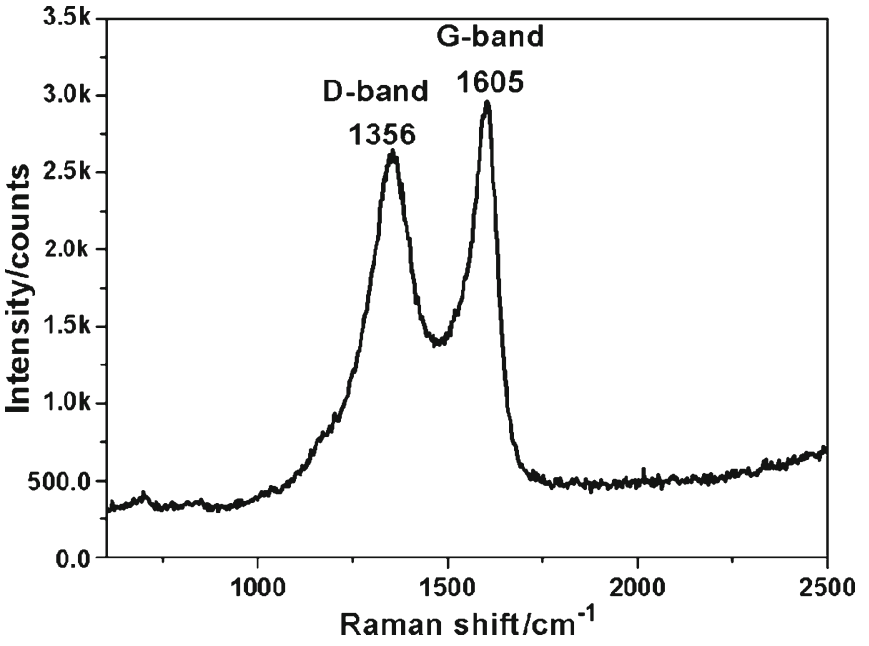

Figure 2. Raman spectrum of VC@C.

Based on the XRD analyses, $\mathrm{V}_{2} \mathrm{O}_{3}$ is an intermediate product for the synthesis of VC. Therefore, the chemical reaction equations of formation of $\mathrm{VC}$ can be expressed as shown in (2) and (3):

$$
\begin{aligned}
& 2 \mathrm{~V}_{3} \mathrm{O}_{7}+5 \mathrm{C} \rightarrow 3 \mathrm{~V}_{2} \mathrm{O}_{3}+5 \mathrm{CO}, \\
& \mathrm{V}_{2} \mathrm{O}_{3}+5 \mathrm{C} \rightarrow 2 \mathrm{VC}+3 \mathrm{CO} .
\end{aligned}
$$

Thus, the total reaction equation of $\mathrm{V}_{3} \mathrm{O}_{7} \cdot \mathrm{H}_{2} \mathrm{O} @ \mathrm{C}$ to prepare VC@C core-shell composite under the thermal treatment condition can be expressed as follows:

$$
\mathrm{V}_{3} \mathrm{O}_{7} \cdot \mathrm{H}_{2} \mathrm{O} @ \mathrm{C} \rightarrow 3 \mathrm{VC} @ \mathrm{C}+7 \mathrm{CO}+\mathrm{H}_{2} \mathrm{O} \text {. }
$$

To reveal the structure of carbon coated on the surface of $\mathrm{VC}$, the corresponding Raman spectrum was carried out, as shown in figure 2 . Two main peaks can be observed. The peaks located at 1356 and $1605 \mathrm{~cm}^{-1}$ are corresponding to the in-plane vibration of disordered amorphous carbon (Dband) and crystalline graphic carbon (G-band), respectively 
which are same as those of amorphous carbon (llie et al 2002; Odani et al 2006; Zhang et al 2011c). The intensity ratio of the G- and D-bands was $I_{\mathrm{G}} / I_{\mathrm{D}}=1.12$ for the as-obtained VC@C compound. The relatively high intensity of the Dpeak further proves that the coating on the surface of $\mathrm{VC}$ comprises disordered carbon. Therefore, the Raman spectrum further confirms that the carbon coating on the surface of $\mathrm{VC}$ is disordered, in agreement with XRD pattern observation.

Further important information about the composition of VC@C composite was collected by EDS, EA and FT-IR. Figure 3 shows typical EDS spectrum of VC@C, which reveals that the surface of the resulting sample consists of only $\mathrm{C}$ and $\mathrm{V}$ elements, in agreement with XRD pattern observation. The content of carbon is 92.40 at $\%$, while vanadium is 7.60 at\%. The atomic ratio of $\mathrm{C} / \mathrm{V}$ in VC@ $\mathrm{C}(\mathrm{C} / \mathrm{V}=$ $12.16)$ is much higher than that of pure $\mathrm{VC}(\mathrm{C} / \mathrm{V}=1)$, which indicates that excess carbon resides on the surface of

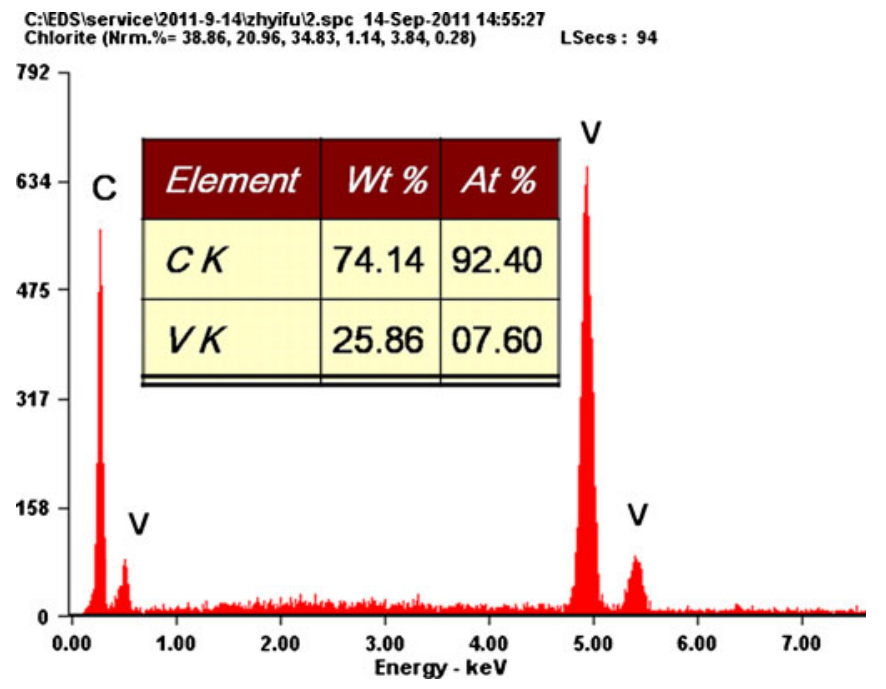

Figure 3. EDS spectrum of VC@C.
VC. Further composition about the carbon on the surface of VC was examined by EA, which shows that the VC@C composite contains $31.09 \mathrm{wt} \%$ of $\mathrm{C}(19.05 \mathrm{wt} \%$ of $\mathrm{C}$ in pure $\mathrm{VC})$ and $1.02 \mathrm{wt} \%$ of $\mathrm{H}$ and the atomic ratio of $\mathrm{C} / \mathrm{H}$ is 2.54 . These results may imply the existence of residual $\mathrm{C}-\mathrm{H}$ groups in the as-obtained VC@C composite after thermal treatment, which can be proved by FT-IR spectrum, as depicted in supplementary data (figure $\mathrm{S} 2$ ). The residual $\mathrm{C}-\mathrm{H}$ groups will facilitate the linkage of catalytic species or polymers to the surface in its potential applications.

Figure 4 shows typical SEM images of the as-obtained VC@C composite. It can be seen from the SEM images that VC@C has belt-like morphology. The length is ranging from $0 \cdot 3 \mu \mathrm{m}$ to several micrometers and the width is about 80 $180 \mathrm{~nm}$ on an average. However, some irregular particles can be observed, which is due to the violent reaction, growth or aggregation by surface and boundary diffusion during the high temperature treatment. In order to get a clear insight into the core-shell structure of the as-synthesized VC@C composite, the corresponding TEM measurement was carried out, as shown in figure 5. The low magnification TEM images (figure 5(a and b)) reveal that the sample consists of a large number of nanotubes, which are carbon nanotubes according to the above analyses and the precursor $\mathrm{V}_{3} \mathrm{O}_{7} \cdot \mathrm{H}_{2} \mathrm{O} @ \mathrm{C}$. These results prove that a new route to synthesize carbon nanotubes consisting of amorphous carbon may be developed and applied in the future. Furthermore, it can be seen from figure 5( $\mathrm{a}$ and $\mathrm{b}$ ), that many $\mathrm{VC}$ particles are encapsulated into the carbon nanotubes. The contrast grade between core (VC) and shell (carbon nanotube) can be clearly observed in figure 5(c). It is to be noted that the morphology of the asprepared VC@C composite is very much different from the morphology of the precursor $\mathrm{V}_{3} \mathrm{O}_{7} \cdot \mathrm{H}_{2} \mathrm{O} @ \mathrm{C}$, as reported in literature (Zhang et al 2011c,d). We suggest that the structure of $\mathrm{V}_{3} \mathrm{O}_{7} \cdot \mathrm{H}_{2} \mathrm{O}$ is completely destroyed and the vanadium oxide severely reacted with carbon or active organic groups (Zhang et al 2011c) to form a new phase VC, accompanying with release of $\mathrm{CO}$. The excess carbon remained to form carbon nanotubes.

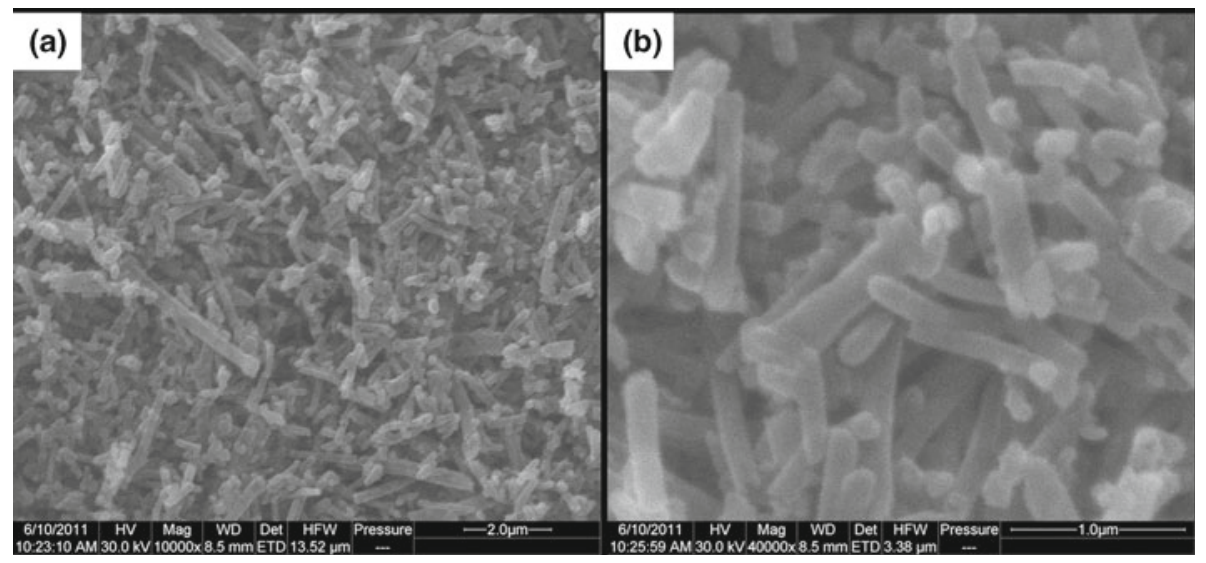

Figure 4. Typical SEM images of VC@C. 


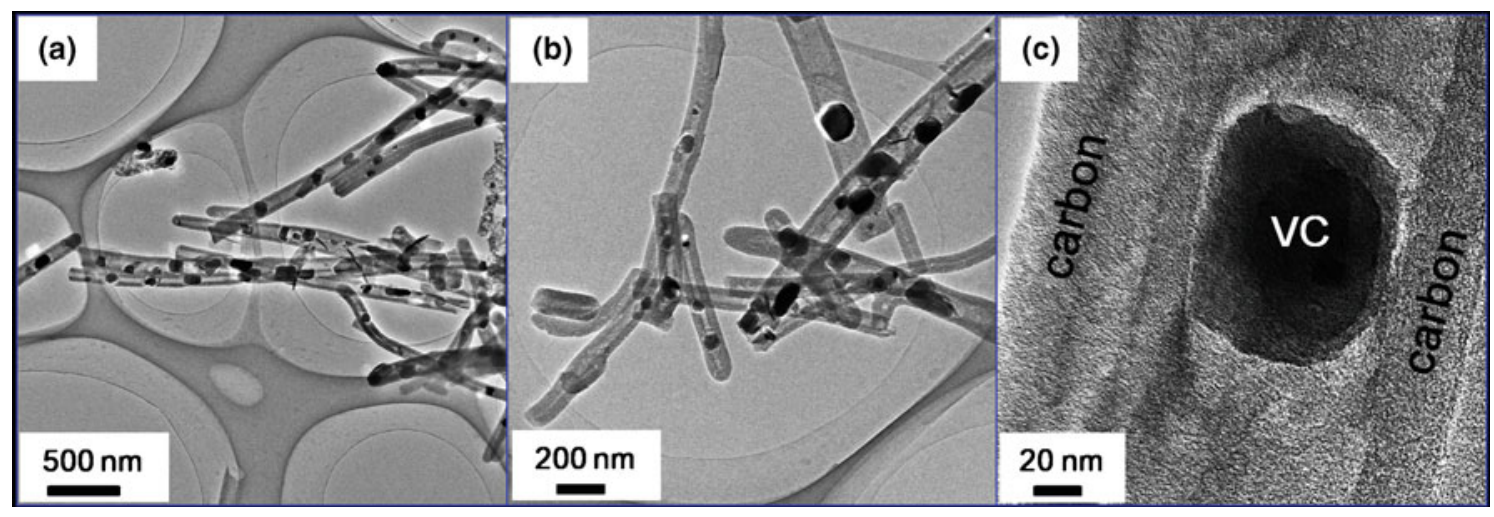

Figure 5. TEM images of VC@C.
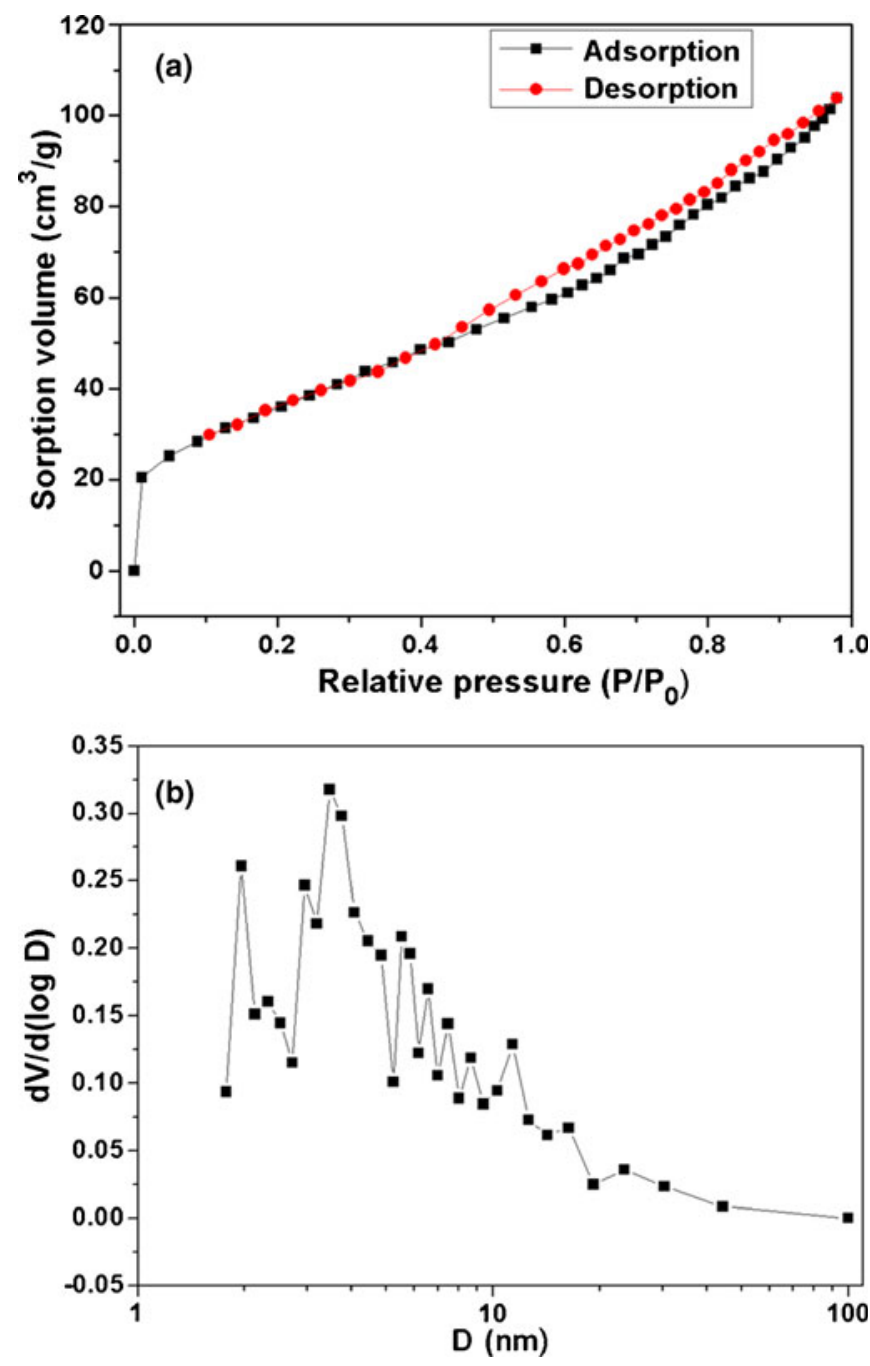

Figure 6. (a) Sorption isotherm of VC@C and (b) pore size distribution data calculated with $\mathrm{BJH}$ method from desorption branch of nitrogen sorption measurements at $77 \mathrm{~K}$.

Based on the above analyses and the chemical reaction (4), some gas was released in the process of preparing VC@C, which might cause lots of pores on the surface of

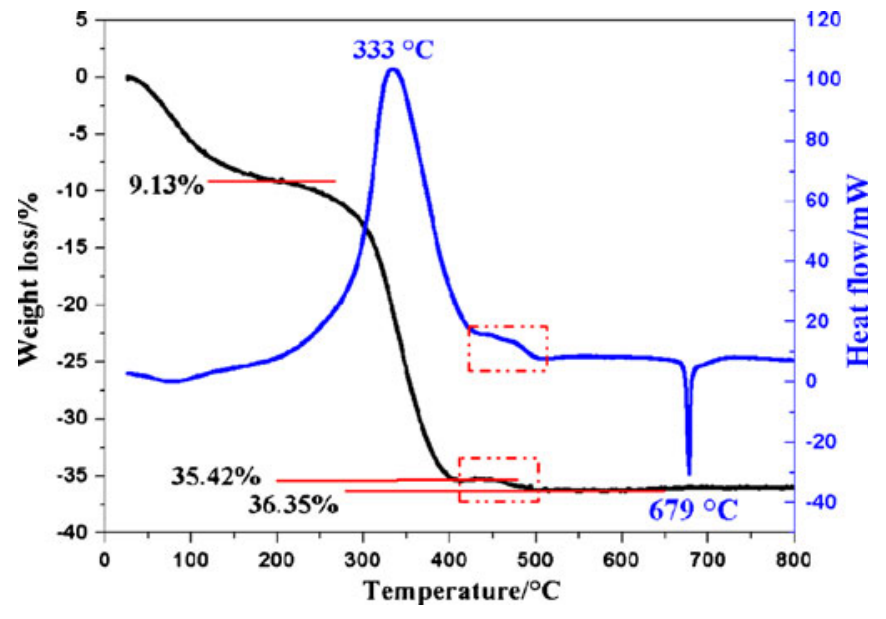

Figure 7. TG and heat flow curves of VC@C in air.

VC@C. These pores can be occasionally observed by TEM images, as shown in figure 5 and clearly seen in supplementary data (figure S3). The above results indicate that the as-synthesized VC@C core-shell structured composite has large specific surface area. Thereafter, the specific surface area and the pore volume of VC@C were measured by BET and BJH methods. BET specific surface area of VC@C is $135.46 \mathrm{~m}^{2} / \mathrm{g}$, which is much bigger than those reported in the literature (Ye et al 2010): 63·36, 31·73, 5.3, 0.9 and $0.3 \mathrm{~m}^{2} / \mathrm{g}$. Nitrogen sorption measurement for VC@C is shown in figure 6. Figure 6(a) shows sorption isotherm of VC@C, revealing that the nitrogen sorption isotherms exhibit the shape of type IV curves (Sing 1982), characteristic of the presence of mesoporous material. Furthermore, the pore size distribution data calculated with the BJH method from the desorption branch of the nitrogen sorption measurements at $77 \mathrm{~K}$ is shown in figure $6(\mathrm{~b})$. The average pore size of the sample is $4.443 \mathrm{~nm}$, which is in the range of the mesoporous material. The measured pore volume is $0.162 \mathrm{~cm}^{3} / \mathrm{g}$.

The thermal behaviour of VC@C was investigated by TG-DTA test, as shown in figure 7. From TG curve, the weight loss is $\sim 9.13 \%$ below $200{ }^{\circ} \mathrm{C}$. The reason can be 
that the water absorbed on the surface of VC@C is releasing or the carbon on the surface can slowly react with $\mathrm{O}_{2}$ in the flowing air. With the increase in temperature, the carbon violently reacts with $\mathrm{O}_{2}$ and releases lot of heat. At about $333^{\circ} \mathrm{C}$, the oxidation is extremely accelerated by large heat evolution, as shown in the heat-flow curve in figure 7 . Besides, the oxidation of VC may occur at this interval. However, both the TG and heat-flow curves have a small platform at about $420^{\circ} \mathrm{C}$, which is due to the oxidation of VC (Ma et al 2009). The weight loss below $420^{\circ} \mathrm{C}$ is $\sim 35.42 \%$ and below $500{ }^{\circ} \mathrm{C}$ is $\sim 36.35 \%$. These results indicate that the oxidation of carbon and VC may be overlapped. In addition, the endothermic peak at $679{ }^{\circ} \mathrm{C}$ is assigned to the melting point of $\mathrm{V}_{2} \mathrm{O}_{5}$, indicating the oxidation product of $\mathrm{VC}$ is $\mathrm{V}_{2} \mathrm{O}_{5}$ in air. Based on above analyses, carbon coated on the surface of $\mathrm{VC}$ has high activity with $\mathrm{O}_{2}$ in air atmosphere, as well as the as-obtained VC@C has large specific surface area confirmed by BET, indicating that the as-prepared VC@C may have high catalytic activity and have potential application in the future.

\section{Conclusions}

In conclusion, a novel approach was successfully developed to fabricate VC@C core-shell structured composite by thermal treatment with the precursor $\mathrm{V}_{3} \mathrm{O}_{7} \cdot \mathrm{H}_{2} \mathrm{O} @ \mathrm{C}$ at $1000{ }^{\circ} \mathrm{C}$ for $2 \mathrm{~h}$ for the first time. It was found that $\mathrm{V}_{2} \mathrm{O}_{3}$ is an intermediate product for the synthesis of $\mathrm{VC}$. The structure of $\mathrm{V}_{3} \mathrm{O}_{7} \cdot \mathrm{H}_{2} \mathrm{O}$ was completely destroyed during the process of formation of $\mathrm{VC}$ and excess carbon remained to form carbon nanotube. The specific surface area, average pore size and measured pore volume of VC@C are $135.46 \mathrm{~m}^{2} / \mathrm{g}, 4.443 \mathrm{~nm}$ and $0.180 \mathrm{~cm}^{3} / \mathrm{g}$, respectively indicating that the as-obtained VC@C composite can be used as a mesoporous material. Furthermore, the thermal behaviour of VC@C composite in air was investigated by TG-DTA test, revealing that the carbon coated on the surface of VC has high activity with $\mathrm{O}_{2}$ in an air atmosphere. The results in this study show that the asprepared VC@C may have high catalytic activity and have potential applications in the future.

\section{Acknowledgements}

This work was partially supported by the fourth installment of Science and Technology Development 2010 Program of Suzhou (SYG201005), the Fundamental Research Funds for the Central Universities, Independent Research Projects of Wuhan University (217274721) and Luojia Young Scholars Program (217273483).

\section{Electronic supplementary material}

Supplementary material pertaining to this article is available on the Bulletin of Materials Science website (www.ias.ac.in/matersci).

\section{References}

Caruso F 2001 Adv. Mater. 1311

Calka A and Kaczmarek W A 1992 Scr. Metall. Mater. 26249

Chen Y J, Zhang H, Ye H N and Ma J H 2011 Int. J. Refract. Met. Hard Mater. 29528

Choi J G 1999 J. Catal. 182104

Dastidar S, Bharath P and Roy A 2011 Mater. Sci. B34 199

Fard H and Baharvandi H 2011 Mater. Sci. B34 883

Frantz P, Didziulis S V, Fernandez-Torres L C, Guenard R L and Perry S S 2002 J. Phys. Chem. B106 6456

Gupta C K and Krishnamurthy N 1992 Extractive metallurgy of vanadium (Elsevier Science \& Technology Books) Ch. 4

Hu Z F, Chen C, Meng H, Wang R H, Shen P K and Fu H G 2011 Electrochem. Commun. 13763

llie A, Durkan C, Milne W I and Welland M E 2002 Phys. Rev. B66 045412/1

Kapoor R and Oyama S T 1995 J. Solid State Chem. 120320

Lee J S, Locatelli S, Oyama S T and Boudart M 1990 J. Catal. 125 157

Li P G, Lei M and Tang W H 2008 Mater. Res. Bull. 433621

Liermann H P, Singh A K, Manoun B, Saxena S K, Prakapenka V B and Shen G 2004 Int. J. Refract. Met. Hard Mater. 22129

Ma J H, Wu M N, Du Y H, Chen S Q, Ye J and Jin L L 2009 Mater. Lett. 63905

Ma S F, Liang J, Zhao J F and Xu B S 2010 Cryst. Eng. Commun. 12750

Nakamura K and Yashima M 2008 Mater. Sci. Eng. B148 69

Odani A, Pol V G, Pol S V, Koltypin M, Gedanken A and Aurbach D 2006 Adv. Mater. 181431

Oelerich W, Klassen T and Bormann R 2001 J. Alloys Compd. 322 L5

Pang W K, Low I M, O'Connor B H, Peterson V K, Studer A J and Palmquist J P 2011 J. Alloys Compd. 509172

Pessall N, Gold R E and Johansen H A 1968 J. Phys. Chem. Solids 2919

Reichl W and Hayek K 2004 J. Catal. 22253

Rodriguez P, Brito J, Albornoz A, Labadi M, Pfaff C, Marrero S, Moronta D and Betancourt P 2004 Catal. Commun. 579

Schwarzkopf P and Kieffer P 1953 Refractory hard metals: borides, carbides, nitrides, and silicides; the basic constituents of cemented hard metals and their use as high-temperature materials (Macmillan Publisher)

Sing K S W 1982 Pure Appl. Chem. 542201

Singh S, Swarnkar R and Gopal R 2010 Mater. Sci. B33 21

Sun X and Li Y 2004 Angew. Chem. Int. Ed. 43597

Sun X M, Liu J F and Li Y D 2006 Chem. Mater. 183486

Venugopalan R and Sathiyamoorthy D 2006 J. Mater. Process. Tech. 176133

Yazawa Y, Furuhara T and Maki T 2004 Acta Mater. 523727

Ye J W, Liu Y, Zhao Z W, Jiang Z T and Tang Z H 2010 J. Alloys Compd. 496278

Yoon S B, Sohn K, Kim J Y, Shin C H, Yu J S and Hyeon T 2002 Adv. Mater. 1419

Zhang B and Li Z Q 2005 J. Alloys Compd. 392183

Zhang Y, Liu X, Xie G, Yu L, Yi S, Hu M and Huang C 2010 Mater. Sci. Eng. B175 164

Zhang Y, Fan M, Niu F, Zhong Y, Huang C, Liu X, Wang B and Li H 2011a Micro. Nano. Lett. 6888

Zhang Y F, Liu X H, Nie J R, Yu L, Zhong Y L and Huang C 2011b J. Solid State Chem. 184387 
Zhang Y F, Liu X H, Chen D Z, Yu L, Nie J R, Yi S P, Li H B and Huang C 2011c J. Alloys Compd. 509 L69

Zhang Y et al 2011d Curr. Appl. Phys. 111159

Zhang Y, Fan M, Niu F, Wu W, Huang C, Liu X and Li H 2012a Curr. Appl. Phys. 12875

Zhang Y, Fan M, Liu X, Xie G, Li H and Huang C 2012b Solid State Commun. 152253

Zhang Y, Fan M, Liu X, Huang C and Li H 2012c Eur. J. Inorg. Chem. 20121650
Zhang Y, Fan M, Wu W, Hu L, Zhang J, Mao Y, Huang C and Liu X 2012d Mater. Lett. 71127

Zhang Y, Zhang F, Yu L, Fan M, Zhong Y, Liu X, Mao Y and Huang C 2012e Colloids Surf. A396 144

Zhao Z W, Zuo H S, Liu Y, Song W Q, Mao S F and Wang Y R 2009 Int. J. Refract. Met. Hard Mater. 27971

Zygmunt J, Krumeich F and Nesper R 2003 Adv. Mater. 15 1538 